\title{
Interleukin-2 Family Members and their Role in Demyelinating Disease
}

\section{Bhopale MK*}

Department of Microbiology, R.D. Gardi Medical College, India
*Corresponding author: Mahendra K. Bhopale, Ph.D., D.Sc, Department of Microbiology \& Director (Research), R.D. Gardi Medical College, Ujjain, MP 456001, India, Tel: +91-7368261235; Email: mbhopale@yahoo.com

\section{Review Article}

Volume 2 Issue 2

Received Date: September 05, 2020

Published Date: September 22, 2020 DOI: $10.23880 /$ aii-16000125

\section{Abstract}

Interleukin-2 (IL-2) has a family which includes IL-2, IL-4, IL-7, IL-9, IL-15 and IL-21 cytokines. This family group of an IL-2 cytokine plays important, but different roles in neurologically related demyelinating disease studied in multiple sclerosis (MS) and it's experimentally induced rodent models. IL-2 play role in strong T-cell expansion and participates in the maintenance of T-regs cells, but also keep in the stimulation and proliferation of pathogenic T cells. IL-4 induces differentiation of naïve helper T cells (Th0) to Th2 cells. IL-7 promotes Th1 cell differentiation. IL-9 is a hematopoietic growth factor for major pathogenic Th17 cells in EAE. IL-15 is necessary for memory CD8+ T cells and plays a negative regulatory role through CD8+ CD122+ T cells in reducing Th17-mediated inflammation. IL-21 has potent regulatory effects on the natural killer (NK) cells and cytotoxic T cells. IL-21 activates CD4+ and up-regulates the Th2 and Th17 subsets of T helper cells. Based on different roles of each family member in demyelinating disease, bio-agents and therapeutic agents have been attempted in an experimental model to study their role in demyelinating disease is described in the present review.

Keywords: Multiple Sclerosis; Experimental Autoimmune Encephalomyelitis; Demyelinating Disease; Role of IL-2, IL-4, IL7, IL-9, IL-15, IL-21 Cytokines; Drugs; Bio-agents; Immune Response; Therapy

Abbreviations: IL-2: Interleukin 2; IL-4: Interleukin 4; IL-7: Interleukin 7; IL-9: Interleukin 9; IL-15: Interleukin 15; IL-21: Interleukin 21; JAK: Janus Kinase; Th Cell: T helper Cell; Treg: Regulatory T Cell; CNS: Central Nervous System; MS: Multiple Sclerosis; EAE: Experimental Autoimmune Encephalomyelitis; IFN- $\gamma$ :Interferon Gamma, MAPK: MitogenActivated Protein Kinase; Ikzf4: IKAROS Family Zinc Finger 4; NK cells: Natural Killer Cells; DAB389IL-2: Diphtheria Toxin IL-2 Fusion Protein; IL2Nag: IL-2/NAg Fusion Protein; STAT: Signal Transducer and Activator of Transcription; aaMphis: Alternatively Activated Macrophages; Foxp3: Forkhead Box P3; BM-MSCs: Bone Marrow Mesenchymal Stem Cells; MMP9: Matrix Metallopeptidase 9; NF-кB: Nuclear Factor KappaLight-Chain-Enhancer of Activated B Cells; Tfh: T Follicular Helper Cells; MBP: Myelin Basic Protein; Ad-MSCs: Adipose Tissue Derived Mesenchymal Stem Cell; mAb: Monoclonal
Antibodies; Jagged2: Jagged Delta-2 (Notch ligands); TM $\beta$ 1 mAb: TM $\beta-1$ Monoclonal Antibody; NR4A2: Nuclear Receptor Subfamily 4 Group A Member 2 (Protein Coding gene); IFN- $\beta$ : Interferon Beta; PTPN22: Protein Tyrosine Phosphatase Non-Receptor Type 22.

\section{Introduction}

Multiple sclerosis (MS) is a demyelinating disease in humans. This disease is an inflammatory disorder of the brain and spinal cord in which focal lymphocytic infiltration leads to loss and dysfunction of myelin and axons. Experimental autoimmune encephalomyelitis (EAE) serves as an animal model of MS, is used to study the clinical and pathological criteria to evaluate the effect of drugs and bio-agents by cytokine changes as potential therapeutic interventions. 
In acute and relapsing MS patients, the disease response is contingent upon activating effector phenotype CD4+ and CD8+ T cells. CD4+CD25hi regulatory $\mathrm{T}$ cells demonstrated due to the CD4 cells IL- 2 responsiveness in demyelinating disease. Interferon- $\gamma$ secreting cells and T-lymphocyte subtypes play an important role in activating interleukin-17 under interleukin-23 control.

Interleukin-2 is a member of a family which includes IL-2, IL-4, IL-7, IL-9, IL-15 and IL-21 [1]. IL-2 cytokine plays an important role in Th1 and Th17 regulation whereas CD4+FoxP3+ regulatory $\mathrm{T}$ cells (Tregs) down-regulate the immune response. MS patients possess either a lower frequency of Tregs or impairment in their suppressor function, promotes disease development [2]. Based on their cytokine production, CD4+ T cells is divided into two helper lineages known as Th1 and Th2 cells. Th1 cells, which produce IFN- $\gamma$, mediate inflammation in the CNS while Th2 cells produce IL-4 in demyelinating disease. IL-2 participates in the maintenance of T-regs cells and reduces self-reactive $\mathrm{T}$ cells. Th17 cells play in the pathogenesis of demyelination is known as Th17/Th1 paradigm [3].

IL-2 has a similar function as IL-15, enhances activity of CD8+ cells and induces CD8+CD44hi memory T cells. IL-15 stimulates B cells and dendritic cells but does not stimulate $\mathrm{T}$ regulatory cells (Tregs). There are primary differences between IL- 2 and other family member like IL-9, which enhance regulatory CD4 (+) FoxP3 (+) T regulatory (Treg) cell survival and T helper 17 (Th17) cell proliferations. On the other hand, IL-21 activates immunoregulatory circuit that is important in the modulation of the course of the autoimmune disease. IL-21 shares sequence homology with IL-2 and the IL-21 receptors contain a gamma-chain common to IL-2. IL-21 also affects the homeostasis of Tregs [4].

It is an important study to understand the mechanisms of immune-mediated destruction or repair CNS components in the experimental models of MS and to provide each IL-2 family member playing an individual role in the immune system in demyelinating disease to present effective design of therapeutics in the review.

\section{Interleukin-2}

Interleukin-2 (IL-2) is a type of cytokine singling molecule in the immune system that regulates the activities of leukocytes and lymphocytes for immunity. The IL-2 receptor complex consists of IL-2 receptor alpha (CD25), IL-2 receptor beta (CD122) and a common gamma chain ( $\gamma \mathrm{c}$ ) (CD132) is shared by all members of this family. IL-2R is comprised of three distinct non-covalently linked chains known as $\alpha$ CD25. IL-2 $\alpha$ subunit does not participate in signaling. In IL-2, beta chain with Janus kinase 1 (JAK1) is capable of adding phosphate groups, whereas gamma chain complexes with tyrosine kinase known as JAK3. CD4+T cells regulate effective immune response to pathogens. Naive CD4+T cells activate after interaction with antigen-MHC complex and differentiate into specific subtypes depending on the cytokine milieu of the microenvironment. Besides the classical T-helper 1 and T-helper 2, other subsets identify as T-helper 17, regulatory $\mathrm{T}$ cell, follicular helper $\mathrm{T}$ cell, and T-helper 9 and each characterize with a cytokine profile [5].

IL-2 plays role in T-cell activation, Treg induction, NKcell activation and DC differentiation. IL-2 activates Th1 and Th17 T-cells, which produce a series of pro-inflammatory cytokines [6]. MAPK pathways regulate $\mathrm{T}$ cell activation, differentiation, effector function, apoptosis and development [7]. Ikzf4 transcription factors, plays an important role in IL-2 and Th17 production by CD4+ T cells and maintains the Treg stability and effector T cell [8].

Regulatory T- cells (Tregs) express IL-2 receptors (IL-2Rs) which play an important role in preventing the development of autoimmunity. IL-2 and Tregs have been demonstrated in eliciting immune response and preventing autoimmunity with neurotrophic factors function in remyelination $[9,10]$. CD25 regulatory $\mathrm{T}$ cells determine secondary remission, but not a primary clinical episode of disease in EAE. The first remission occurred even after depletion of Treg cells, but secondary remissions from EAE are ablated [11]. It is known that IL-17-producing CD4 cells in the CNS have the pathogenic role of an independent (nonTh1) IL-17-producing pro-inflammatory effector T cells and IL-17-producing pro-inflammatory effector T cell class in EAE [12]. On the other hand, B cells participate in the regulation of CNS autoimmune disease through the production of autoantibodies. Adoptive transfers of myelin-specific CD4+ Th1 cells into naïve recipient mice promote the induction of EAE $[13,14]$.

IL-2 role in therapy IL-2 recombinant fusion protein, known as a $\mathrm{DAB}_{389} \mathrm{IL}-2$ target IL-2R bearing CD4+ cells is attempted in EAE rodent models. $\mathrm{DAB}_{389} \mathrm{IL}-2$ suppresses at the early time points, but negative consequences at later time points. $\mathrm{DAB}_{389} \mathrm{IL}-2$ directly targets $\mathrm{CD}^{+}$, and $\mathrm{CD}^{2} 5^{+}$, IL-2R T cells and effector T cell function $[15,16]$. DAB ${ }_{389} \mathrm{IL}^{\mathrm{L}-2}$ indirectly suppresses the activation of macrophage CD169+ (ED3+) and microglia CD11b/c (OX42+) populations in the CNS. $\mathrm{DAB}_{389} \mathrm{IL}^{-2}$ also reduce $\mathrm{CD} 3^{+} \mathrm{CD} 4^{+}, \mathrm{CD}^{+} \mathrm{CD}^{+}, \mathrm{CD}^{+}{ }^{+} \mathrm{CD} 8^{+}$, $\mathrm{CD}^{+} \mathrm{IL}^{-} 2^{+}, \quad \mathrm{CD} 3^{+} \mathrm{IFN}-\gamma^{+}, \quad \mathrm{CD}^{+} \mathrm{TNF}^{-} \alpha^{+}, \quad \mathrm{CD}^{+} \mathrm{CD}^{+} 5^{+} \quad$ cell subpopulations $[17,18]$. However, $\mathrm{DAB}_{389} \mathrm{IL}-2$ also exhibit toxic side effect. IL-2-caspase-3, another chimeric protein, is designed to target and kill cells expressing the high affinity IL-2 receptor. IL-2-caspase-3 chimeric protein treatment to EAE-induced mice results a significant delay in disease with a reduction in disease symptoms [19]. 
There is another fusion protein known as interleukin-2/ neuroantigen (NAg) fusion protein is studied in the EAE model. IL2NAg (IL-2/NAg fusion protein) injection in Lewis rats results in suppression of encephalitogenic disease. It shows an increased Tregs activity with low-dose IL-2 immunotherapy that prevents the development of T-follicular helper CD4+ T-cell subset promoting long-term effector B-cell responses in autoimmune disease patients [20]. Daclizumab is a humanized monoclonal antibody CD25 alpha subunit of the high-affinity interleukin (IL)-2 receptor, reduce IL-2mediated lymphocyte activation and up regulation of CD56bright natural killer cells in relapsing-remitting multiple sclerosis [21].

FSD-C10 is a Fasudil derivative which reduces the severity through the modulation of the immune response by induction of neuroprotective molecules in the CNS in EAE. Intranasal delivery of FSD-C10 effectively ameliorates the clinical severity, inflammatory infiltration in the CNS and such treatment effectively induced $\mathrm{CD} 4^{+} \mathrm{CD} 25^{+} \mathrm{FOXP} 3^{+}$regulatory $\mathrm{T}$ cells. FSD-C10, thus, delays the onset and promotes neuro protection in EAE [22]. T cell cytokine production alteration has been observed in EAE mice by using immunostimulatory CpG oligonucleotide. CpG can have different effects on T cell cytokine production depending on whether they are present in the priming phase or during the ongoing autoimmune response, the effects differ qualitatively and quantitatively in the inflamed CNS [23]. B cells participate in the pathogenesis and regulation of CNS autoimmune disease has been found through the production of auto-antibodies. The auto antigenspecific B cell lineage leads to production of the pathogenic autoantibodies. These auto antigen-specific B cells have been consistently identified in the circulation with high serum levels of auto antigen-specific antibodies [24].

Other reports show that interleukin-2 (IL2) repressor genes ZEB1 influence $T$ cell regulation. ZEB1 affect the regulation, novel pathways to contribute pathogenesis and inflammation in EAE [25]. Neuropilin-1 (Nrp1) has been implicated in several aspects of immune function, including maintenance of the immune synapse and development of regulatory Treg cells [26]. CD4+ T-cell activates trafficking regulating by Itk signals and promotes neuroinflammation. Itk $^{-/-} \mathrm{CD} 4^{+} \mathrm{T}$ cells show significant reduction in the production of T-helper 1 (Th1) and Th17 cytokines [27]. Immune modulation of IL-2 receptor restores impaired immune regulation by increasing the proportion of CD155-expressing CD4 (+) T cells and the cytolytic activity of NK cells in MS [28].

\section{Interleukin-4}

The interleukin 4 (IL-4) is a cytokine that induces differentiation of naïve helper cells (Th0) to Th2 cells.
IL- 4 receptor composed of IL- $4 \alpha$ of a subunits bind with a common $\gamma$ chain. IL-4 activate by the JAK1 and JAK3 signaling transducer of STAT6. IL-4 receptor-mediated STAT5 activation is dependent on the presence of $\gamma c$ and JAK3 receptor complex. IL-4 decreases the production of Th1 cells, macrophages, IFN- $\gamma$ and dendritic IL-12.

CNS-derived IL-4 is a critical regulator in mice with a deficiency in IL-4 production in the CNS with a significant increase in the absolute number of infiltrating inflammatory cells. It induces a state of alternative activation in microglial cells [29]. CNS-resident microglial cells in both the resting and activated state produced the protein $\mathrm{Ym} 1$, which is a marker of alternatively activated macrophages in an IL-4dependent manner. Th2 cytokines IL-4 and IL-5 regulate serum production, which effects on regulatory $\mathrm{T}$ cell expression of Foxp3 and suppressing the production of proinflammatory cytokines in EAE [30].

IL-4 role in therapy There are studies related IL-4 involvement in therapy. For example, the combination of methylprednisolone (MP) and human bone marrowderived mesenchymal stem cells (BM-MSCs) reduce proinflammatory cytokines (IFN- $\gamma$, TNF- $\alpha$, IL-17) and enhanced anti-inflammatory cytokines (IL-4, IL-10). Treatment with dimethyl fumarate (DMF) reduces the severity of EAE in mice and the relapse rates of MS in humans. DMF promotes an IL$23^{\text {low }} \mathrm{IL}-12^{\text {low } I L-10^{+}} \mathrm{DC}$ phenotype and its efficacy is mainly explained by a suppression of encephalitogenic Th17/Th1 cells and an induction of IL-4+Th2 cells. Dimethyl fumarate promotes an IL-17 $\mathrm{A}^{\text {low } I F N-} \gamma^{\text {low } I L-} 4^{+} \mathrm{CD} 4^{+} \mathrm{T}$ cell phenotype. DMF-treated myelin peptide-reactive IL-17A ${ }^{\text {low }} I F N-\gamma^{\text {low }} I L-$ $4^{+} \mathrm{CD} 4^{+} \mathrm{T}$ cells prior to immunization in EAE reduce the severity of encephalomyelitis [31]. The drug, Ustekinumab (UTZ) (Stelera®), oral administration decreased proinflammatory cytokines Th1-like cytokines IL-2, IL-12, IFN- $\gamma$, IL-17 (Teff) and increased counter-regulatory cytokines IL4, IL-10 and IL-13 in recipients of donor cells from UTZ fed mice [32]. Another drug, N1rp12 plays a protective role by suppressing inflammation altered $N F-\kappa B$ regulation and IL-4 production during the development of EAE [33,34].

The suppressive effect of myelin basic protein (MBP) peptide 68-86 in the EAE Lewis rats, ameliorate the disease with decreased infiltration of ED1+ macrophages and CD4+ $\mathrm{T}$ cells within the central nervous system [35]. Interestingly, mesenchymal stem/stromal cells (MSCs) therapy has considerable promise for tissue regeneration. MSCs-IFN $\beta$ reduces migration into the inflammatory cells in the CNS of EAE mice. Bone marrow-derived mesenchymal stem cells (BM-hMSCs) induce Th2-polarized immune response interferon gamma (IFN- $\gamma$ ) producing Th1 cells and IL17 producing Th17 inflammatory cells increases in IL-4 producing Th2 cells [36-38]. 


\section{Interleukin-7}

Interleukin-7 (IL-7) cytokine plays an important function in T-cell development and memory cell induction. IL-7 is a hematopoietic growth factor, secreted by stromal cells in the red marrow and thymus. It is also produced by keratinocytes, hepatocytes, neurons, endothelial cells but not by lymphocytes. IL-7 stimulates and differentiate pluripotent hematopoietic stem cells proliferate all lymphoid lineage B cells, T cells and NK cells. IL-7 is genetically associated with susceptibility to demyelinating disease in multiple sclerosis. IL-7R consists of two subunits, interleukin-7 receptor- $\alpha$ (CD 127) and common- $\gamma$ chain receptor (CD 132). The IL-7/IL7R signaling axis perturbation is associated with enhanced susceptibility to MS. IL-7 is an essential for survival of pathogenic Th17 cells. IL-7R antagonism differentiates Th17 cells through the inhibition of JAK-STAT5 pathways, brings alteration in Bcl-2 and Bax proteins, which decrease severity of disease in EAE. The role of IL-7-IL-7R in Th17 cell survival implicates in the treatment of autoimmune disease [39].

A progressive enrichment for CD4 single-positive Foxp3 (+) regulatory $\mathrm{T}$ cells is characterized by accelerating differentiation and proliferation of regulatory $\mathrm{T}$ (Treg) cells. Stromal cells increase expression through a mechanism involves IL-7. IL-17-producing CD4 (+) T (Th17) cells, along with IFN- $\gamma$-expressing Th1 cells, represent two major pathogenic T cell subsets in EAE. There is a link between IL23-driven pathogenic T cells and IL-7/IL-7R signaling. IL-7 inhibits the differentiation of Th17 cells.

IL-7 role in therapy IL-7 $\alpha$ alters $\mathrm{T}$ cell and nonhematopoietic cell lineages response in EAE. IL7R $\alpha$ expression is identified on astrocytes and oligodendrocytes endogenous to the CNS. IL- $7 \alpha$-blocking antibodies before or after onset of paralysis exhibits reduction in clinical signs and also activation of regulatory $\mathrm{T}$ cells and $\mathrm{B}$ cells, and natural killer cell with markedly reduces lymphocyte infiltration into the central nervous system confers Th1/Th17 responses in the EAE [40]. IL-7 activates GM-CSF produces CD4+ T cells by STAT 5 to induce more severe EAE than Th17 or Th1 cells [41]. The effect of CD127 blockade has also been observed in an inbred/SPF mouse EAE model in a positive response to the treatment. IL-7 signaling is a prerequisite for optimal $\mathrm{CD}^{+} \mathrm{T}$ cell activation that IL-7R antagonism effective in treating $\mathrm{CD}^{+} \mathrm{T}$ cell-mediated neuroinflammation $[42,43]$. MS patients have lower serum IL-7 and a higher membrane IL-7R $\alpha$ expression in CD56bright NK cells [44]. IL-7 bioactivity provides a basis to explain the increased risk of autoimmunity genotype-induced elevations of sIL7R $\alpha$ [45]. Serum profile of high IL-7 signifies a TH1-driven form of MS in IFN- $\beta$ therapy and blockade of IL-7 and the IL-7R $\alpha$ pathway have therapeutic potential in MS and other autoimmune diseases [46]. Blocking IL-7 signaling in myelin-specific
CD4 T cells by $\alpha$ IL-7R $\alpha$ significantly delays EAE onset and reduces disease severity. The IL-7R $\alpha$ expression suppresses the encephalitogenic potential of myelin-specific CD4 T cells and has therapeutic benefits. IL-7R $\alpha$ confers susceptibility by influencing autoimmune Th1/Th17 responses in the EAE model $[47,48]$.

Interferon- $\beta$ is the major treatment for multiple sclerosis (MS). However, this treatment is not always effective. Here we see congruence in outcome between responses to IFN- $\beta$ in experimental autoimmune encephalomyelitis (EAE) and relapsing-remitting MS (RRMS). IFN- $\beta$ is effective in reducing EAE induced by $\mathrm{T}_{\mathrm{H}} 1$ cells, but exacerbated disease induced by $T_{H}$ 17. $T$ helper 1 ( $\left.T_{H} 1\right)$-driven, but not a TH17-driven, form of MS exhibited a good clinical response to interferon- $\beta$ (IFN- $\beta$ ) therapy. Blocking IL-7 signaling in myelin-specific CD4 T cells by $\alpha$ IL-7R $\alpha$ significantly delays EAE onset and reduces disease severity $[49,50]$.

\section{Interleukin-9}

Interleukin 9 (IL-9) cytokine plays important role in T-cell activation and mast cell activation. IL- 9 is a dominant cytokine in Th9 cells, has been proven to play a pathogenic role in the EAE model by augmenting $\mathrm{T}$ cell activation and differentiation. The IL- 9 receptor complex is shared by $\gamma$ subunit of IL-2R $\gamma$. The ligand receptor binding of IL-9 leads to the activation of various JAK kinase and STAT proteins for different biological responses [51]. IL-9 is a cell signaling protein molecule encoded by CD4+ helper cells. IL-9 is a hematopoietic growth factor as a mediator of Th17 cells. IL-9 receptor (IL-9R) is highly expressed in astrocytes, oligodendrocyte progenitor cells, oligodendrocytes and microglia cells in the brain and spinal cord during EAE [52]. In MS and EAE, microglia induce different effector functions that can be both neuroprotective and detrimental. Currently, it is believed that the initial response of microglia is beneficial, but the chronic activation of microglia contributes to neurodegeneration [53].

Myelin oligodendrocyte glycoprotein (MOG)-specific effector T cell subsets (Th1, Th17 and Th9) can induce EAE independently of each other. They all produce different cytokines and the inflammatory process advances. However, the clinical disease manifestations are indistinguishable [54]. A new effector $\mathrm{T}$ subset Th9 cells are identified as myelin oligodendrocyte glycoprotein-specific Th1, Th17, and Th9 but not Th2 cells in EAE upon adoptive transfer. Th9 cells are a newly discovered $\mathrm{CD} 4^{+} \mathrm{T}$ helper cell subtype, characterized by high interleukin (IL)-9 secretions. Growing evidences suggest that Th9 cells participate in the pathogenic mechanism of multiple sclerosis (MS). There is dynamic "crosstalk" between Th9 and mast cells. IL-9-mast cell axis in EAE also determines its interaction after neutralizing 
anti-IL-9 antibody. IL-9 blockade using anti-IL-9 antibody treatment ameliorates EAE with decreasing in mast cell infiltration in CNS [55].

IL-9 role in therapy IL-9 is seen in the context of $\mathrm{T}_{\mathrm{h}} 2$ associated inflammatory conditions in allergic inflammation. IL-9 is considered just another $\mathrm{T}_{\mathrm{h}} 2$ cytokine and thought to be redundant among other $\mathrm{T}_{\mathrm{h}} 2$ cytokines (i.e. IL-4, IL-5 and IL-13). IL-9 seems to be one cytokine of many sources, and therefore, interest in IL-9 biology and in its significance. The role of $T_{h} 9$ supporting a role for $T_{h} 9$ cells in EAE, suggesting that targeting $\mathrm{T}_{\mathrm{h}} 9$ cells may provide an additional approach in the treatment of such autoimmune conditions. There are other conditions where promotion of $\mathrm{T}_{\mathrm{h}} 9$ cells may therapeutically beneficial.

The protective effect of anti-IL-9 mAb treatment in EAE is mediated not only via suppression of IL-9-induced inflammatory reactions, but also via inhibition of the induction of $\mathrm{MOG}_{35-55-}$ peptide-specific Th17 and Th1 cells, which in turn leads to reduced infiltration of T cells into the CNS. This study evidence for an important pathogenic role of IL-9 in EAE and also indicate that anti-IL-9 mAb treatment represent a therapeutic agent. Further, anti-IL-9 mAb immunization with myelin proteolipid protein (PLP180199) peptide in Complete Freund's Adjuvant (CFA) exhibit considerably fewer infiltrating immune cells in the CNS and also suppress IL-17 and IFN- $\gamma$ expression. IL-9 affects Th17 cells not only through the activation of STAT3, but also STAT1, suggesting that IL-9 triggers complex STAT signaling pathways. IL-9 control pathways central to the induction and development of EAE whereas disruption of the IL-9 gene reduces inflammatory infiltrates in the CNS and attenuates clinical symptoms of EAE [56].

\section{Interleukin-15}

Interleukin-15 (IL-15) is a cytokine with structure similar to IL-2. IL-15 is an inflammatory cytokine. IL-15 binds through the signaling pathway of IL-15R $\alpha$ receptor and IL-15 $\beta$ subunit through the activation of Jak1 and Jak2 in $\gamma$ c subunit with the help of STAT3 and STAT5. The role of IL-15 is STAT5 activation and IL-17A production in CD4 T lymphocytes. Th17 cells play critical roles as a negative regulatory role in reducing Th17-mediated EAE inflammation through $\mathrm{CD}^{+}$CD122+ $\mathrm{T}$ cells [57].

The role of T regulatory cells $\left(\mathrm{T}_{\text {reg }}\right)$ has been demonstrated to be capable of controlling CNS autoimmunity in several EAE models. Transfer of CD25+ $\mathrm{T}_{\text {reg }}$ ameliorated EAE symptoms. In addition, non-specific ablation of natural $\mathrm{T}_{\text {reg }}$ by antiCD25 antibodies has been reported to exacerbate EAE. Furthermore, $\mathrm{T}_{\text {reg }}$ have been shown to prevent spontaneous EAE development or delay spontaneous EAE onset [58].
This indicates that IL-15 has a negative regulatory role in fine-tuning of IL-17A production and Th17-mediated inflammation.

$I L-15$ role in therapy Interleukin (IL)-15 can cross the blood-brain barrier to act on its specific brain receptor (IL15R $\alpha$ ) and co-receptors. The important roles of neuronal IL-15 and IL15R $\alpha$ in EAE are suggested by the up-regulation of IL15R $\alpha$ in different regions of the brain and spinal cord. Contrary to expectations, IL-15 treatment lessened EAE severity. IL-15 knockout mice showed heightened susceptibility to EAE with significantly higher scores that were decreased by treatment with IL-15. IL-15 improves the CNS autoimmune disorder as a potential therapeutic agent.

Interleukin 15 (IL-15) expression induces the secretion of inflammatory cytokines, inhibits the apoptosis of activated $\mathrm{T}$ cells and prolongs the survival of $\mathrm{CD}^{+}$memory $\mathrm{T}$ cells. An IL-15 isoform is a natural antagonist for IL-15 function demonstrated that IL-15 $\Delta \mathrm{E} 6$ activation and function as a negative feedback mechanism to IL-15-mediated inflammatory events [59]. Deubiquitinating enzymes (DUBs) studies have shown emerging as crucial regulators of EAE and MS. Daclizumab, an antibody against the IL-2R $\alpha$ chain, inhibits brain inflammation in MS [60]. IL-15 improves the CNS autoimmune disorder with a negative regulatory role of IL-17A production. Blockade of IL-15 signaling by TM $\beta$ 1 monoclonal antibody (mAb) treatment aggravates EAE severity, suggest that IL-15 activate through CD8+ CD122+ $\mathrm{T}$ cells, has a negative regulatory role in reducing IL-17 production $[61,62]$.

\section{Interleukin-21}

Interleukin-21 (IL-21) is a member of the common gamma-chain-dependent IL-2 cytokine family. IL-21 is a key modulator of lymphocyte development, proliferation, and differentiation. IL-21 is highly expressed in activated CD4 (+) T cells. IL-21r is structurally similar into the receptors for IL$2 \mathrm{R}$ or IL-15 binding with the common $\gamma \mathrm{c}$ chain to IL-21. It plays a critical role in the expansion and differentiation of the Th17 cell subsets [63].

IL-21 is an autocrine amplification factor, essential for differentiation of Th17 cells, but it does not protect actively induced EAE mice by blocking IL-21 or IL-21 receptor. Th17 cells do transfer EAE but whether the disease is dependent on IL-17 produced by the Th17 cells is not known. It should be noted that the pathogenic capacity of Th17 cells cannot be reduced due to the effects of IL-17 alone but the effects of all cytokines secreted by the Th17 cell subset. Auto reactive purified $\mathrm{T}$ cells from IL-21-treated mice, on transfer cells cause more severe EAE than did the control encephalitogenic T cells [64]. NR4A2 appears to control Th17 differentiation 
and so plays an essential role in the development of Th17mediated autoimmune disease. As NR4A2 is also upregulated during human autoimmune disease, targeting NR4A2 may provide a new therapeutic approach in treating autoimmune disease. NR4A2 controls Th17 differentiation and plays an essential role in the development of Th17mediated autoimmune disease in EAE [65].

The IL-21 receptor (IL-21R) consists of a unique subunit and a common $\gamma$ chain $(\gamma c)$ that is shared with other cytokines of IL-2, IL-4, IL-7, and IL-15. The interaction between IL-21 and IL-21R results in significant effects on both innate and adaptive immune responses. The impact on EAE initiation by IL-21R deficiency was associated with a defect of CD4(+) CD25(+) $\mathrm{T}$ regulatory $\left(\mathrm{T}_{\text {reg }}\right)$ cells and a down-regulated expression of Foxp3 [66]. Studies on IL-17 demonstrate $\gamma \delta$ $\mathrm{T}$ cells, an important source of IL-17 and IL-21 that helped amplify IL-17 production by Th17 cells in autoimmune diseases. IL-17-secreting CD4 T cells (Th17 cells) and IL-17secreting $\gamma \delta$ T cells play a critical pathogenic role in central nervous system (CNS) inflammation in EAE and MS [67].

IL-21role in therapy IL-21 is an essential autocrine amplification factor for differentiation of Th17 cells, the loss of IL-21 or IL-21 receptor (IL-21R) does not protect mice from actively induced EAE. It was demonstrated that DNA vaccination protects from proinflammatory Th17 cell responses during induction of EAE. The mechanism involves IL-17 responses are rescued by silencing of IFN- $\beta$ during DNA vaccination [68].

Proteoglycans (PGs) are complex glycohydrates proteins. Daily oral administration of PG attenuates the clinical and histological severity in a dose-dependent manner in EAE. Salmon cartilage PG attenuates the severity of EAE by suppressing the differentiation of Th17 linage and enhancement of $\mathrm{T}_{\text {reg }}$ expansion. It has potential involvement of activated $\mathrm{T}_{\mathrm{FH}}{ }^{-}$and $\mathrm{B}$-cells for the treatment of progressive MS [69]. There is also an interesting study on helminth protein vaccine combined with FK506 induces Tfh cell for stimulating humoral immune responses inducing long-lived humoral immunity. FK506 is an adjuvant for recombinant protein augments the induction of Tfh cells that express IL-21 and IL-4 and produce B cells. FK506 is a widely used immunosuppressant for treating allergies, autoimmune diseases. FK506 as adjuvant of DNA vaccines induced regulatory $\mathrm{T}$ cells $\left(\mathrm{T}_{\text {reg }}\right)$ and can prevent $\mathrm{MS}$ and other autoimmune disease [70].

\section{Conclusion}

IL-2 cytokine family members play different roles in demyelinating disease studied in EAE rodent models and in MS patients. Such diversity in role and function keep the immune system changes by developing inflammation and demylination in the CNS in the initial phase of the disease and back to the normal in the recovery phase. As many therapies are advancing in demyelinating disease, more study is needed to obtain a comprehensive understanding of the entire process, crucial steps and specific time windows for effective therapeutic interventions to suppress disease before reaching at the advance stage. Here are those studies mentioned, including the role of IL-2, IL-4, IL-7, IL-9, IL-15 and IL-21 played in therapy. The key cytokine roles which govern in therapies make a change in Treg cells and Th17/ Th1 paradigm, which allow selecting a choice in demylinating disease therapy.

\section{Acknowledgement}

Gratefully acknowledged to Dr. V. K. Mahadik, Director, R. D. Gardi Medical College for the encouragements, and to establish and develop research in biomedical sciences.

\section{References}

1. Liao W, Lin JX, Leonard WJ (2011) IL-2 Family Cytokines: New Insights into the Complex Roles of IL-2 as a Broad Regulator of $\mathrm{T}$ helper Cell Differentiation. Curr Opin Immunol 23(5): 598-604.

2. Leung S, Liu X, Fang L, Chen X, Guo T, et al. (2010) The cytokine milieu in the interplay of pathogenic Th1/Th17 cells and regulatory $\mathrm{T}$ cells in autoimmune disease. Cell Mol Immunol 7(3): 182-189.

3. Rostami A, Ciric B (2013) Role of Th17 cells in the pathogenesis of CNS inflammatory demyelination. J Neurol Sci 333(1-2): 76-87.

4. Piao WH, Jee YH, Liu RL, Coons SW, Kala M, etal. (2008) IL21 modulates CD4+CD25+ regulatory T-cell homeostasis in experimental autoimmune encephalomyelitis. Scand J Immunol 67(1): 37-46.

5. Luckheeram RV, Zhou R, Verma AD, Xia B (2012) CD4+T cells: differentiation and functions. Clin Dev Immunol 2012: 925135.

6. Ye C, Brand D, Zheng SG (2018) Targeting IL-2: an unexpected effect in treating immunological diseases. Signal Transduct Target Ther 3: 2.

7. Qi M, Elion EA (2005) Kinase pathways. J Cell Sci 118: 3569-3572.

8. Gokhale AS, Gangaplara A, Lopez-Occasio M, Thornton AM, Shevach EM (2019) Selective deletion of Eos (Ikzf4) in T-regulatory cells leads to loss of suppressive function and development of systemic autoimmunity. J 
Autoimmunity 105: 102300.

9. Moon BI, Kim TH, Seoh JY (2015) Functional Modulation of Regulatory T Cells by IL-2. PLoS One 10(11): e0141864.

10. Linker R, Gold R, Luhder F (2009) Function of neurotrophic factors beyond the nervous system: inflammation and autoimmune demyelination. Crit Rev Immunol 29(1): 43-68.

11. Gärtner D, Hoff H, Gimsa U, Burmester GR, BrunnerWeinzierl MC (2006) CD25 regulatory T cells determine secondary but not primary remission in EAE: impact on long-term disease progression. J Neuroimmunol 172(12): 73-84.

12. Hofstetter HH, Toyka KV, Lehmann MT, Lehmann PV (2007) Kinetics and organ distribution of IL17-producing CD4 cells in proteolipid protein 139151 peptide-induced experimental autoimmune encephalomyelitis of SJL mice. J Immunol 178(3): 13721378.

13. Sokolov AV, Shmidt AA, Lomakin YA (2018) B cell Regulation in autoimmune diseases. Acta Naturae 10(3): 11-22.

14. Rangachari M, Kuchroo VK (2013) Using EAE to better understand principles of immune function and autoimmune pathology. J Autoimmunity 45: 31-39.

15. Phillips SM, Bhopale MK, Constantinescu CS, Ciric B, Hilliard B, et al. (2007) Effect of $\mathrm{DAB}_{389} \mathrm{IL}-2$ immunotoxin on the course of experimental autoimmune encephalomyelitis in Lewis rats. J Neurol Sci 263(1-2): 59-69.

16. Phillips SM, Bhopale MK, Hilliard B, Zekavat SA, Ali MA, et al. (2010) Suppression of murine experimental autoimmune encephalomyelitis by interleukin-2 receptor targeted fusion toxin, DAB(389)IL-2. Cell Immunol 261(2): 144-152.

17. Bhopale MK, Hilliard B, Constantinescu CS, Fujioka $\mathrm{T}$, Ventura E, et al. (2014) $\mathrm{DAB}_{389} \mathrm{IL}-2$ suppresses autoimmune inflammation in the CNS and inhibits T cellmediated lysis of glial target cells. Exp Mol Pathol 96(1): 108-117.

18. Bhopale MK, Hilliard B, Constantinescu CS, Phillips SM, Rostami A (2017) $\mathrm{DAB}_{389}$ IL-2 recombinant fusion toxin effect on lymphocyte-and macrophage-producing cytokine subpopulation cells in experimentally induced demyelinating disease in mice. Immunopharmacol Immunotoxicol 39(6): 318-329.

19. Irony-Tur-Sinai $M$, Lichtenstein $M$, Brenner $T$,
Lorberboum-Galski H (2009) IL2-caspase3 chimeric protein controls lymphocyte reactivity by targeted apoptosis, leading to amelioration of experimental autoimmune encephalomyelitis. Int Immunopharmacol 9(10): 1236-1243.

20. Ballesteros-Tato A, (2014) Beyond regulatory T cells: the potential role for IL-2 to deplete T-follicular helper cells and treat autoimmune diseases. Immunother 6(11): 1207-1220.

21. Derwenskus J, Lublin FD (2014) Future treatment approaches to multiple sclerosis. Handb Clin Neurol 122: 563-577.

22. Li YH, Yu JZ, Liu CY, Zhang H, Zhang HF, et al. (2014) Intranasal delivery of FSD-C10, a novel Rho kinase inhibitor, exhibits therapeutic potential in experimental autoimmune encephalomyelitis. Immunology 143(2): 219-229.

23. Smolianov V, Dehmel T, Vollmar $\mathrm{P}$, Mausberg AK, Kieseier BC, et al. (2011) Alteration of T cell cytokine production in PLPp-139-151-induced EAE in SJL mice by an immunostimulatory $\mathrm{CpG}$ oligonucleotide. J Neuroinflammation 30(8): 59-68.

24. Sun B, Ramberger M, O'Connor KC, Bashford-Rogers RJM, Irani SR (2020) The B cell immunobiology that underlies CNS autoantibody-mediated diseases. Nature Rev Neurol 16(9): 481-492.

25. Stridh P, Hedreul TM, Beyeen AD, Adzemovic MZ, Laaksonen H, et al. (2010) Fine-mapping resolves Eae23 into two QTLs and implicates ZEB1 as a candidate gene regulating experimental neuroinflammation in rat. PLoS One 5(9): e12716.

26. Solomon BD, Mueller C, Chae WJ, Alabanza LM, Bynoe MS (2011) Neuropilin-1 attenuates autoreactivity in experimental autoimmune encephalomyelitis. Proc Natl Acad Sci USA 108(5): 2040-2045.

27. Kannan AK, Kim DG, August A, Bynoe MS (2015) Itk signals promote neuroinflammation by regulating CD4+ T-cell activation and trafficking. J Neurosci 35(1): 221233.

28. Gross CC, Schulte-Mecklenbeck A, Rünzi A, Kuhlmann T, Posevitz-Fejfár A, et al. (2016) Impaired NK-mediated regulation of T-cell activity in multiple sclerosis is reconstituted by IL-2 receptor modulation. Proc Natl Acad Sci USA 113(21): 2973-2982.

29. Ponomarev ED, Maresz K, Tan Y, Dittel BN (2007) CNSderived interleukin-4 is essential for the regulation 
of autoimmune inflammation and induces a state of alternative activation in microglial cells. J Neurosci 27(40): 10714-10721.

30. Soria JA, Arroyo DS, Gaviglio EA, Rodriguez-Galan MC, Wang JM, et al. (2011) Interleukin 4 induces the apoptosis of mouse microglial cells by a caspasedependent mechanism. Neurobiol Dis 43(3): 616-624.

31. Bruck J, Glocova I, Geisel J, Kellerer C, Röcken M, et al. (2018) Dimethyl fumarate-induced IL-17 low IFN- $\gamma$ low IL-4 + Th cells protect mice from severe encephalomyelitis. Eur J Immunol 48(9): 1588-1591.

32. Brod SA (2016) Ingested (oral) anti-IL-12/23 inhibits EAE. J Neurol Sci 361: 19-25.

33. Gharagozloo M, Mahvelati TM, Imbeault E, Gris P, Zerif E, et al. (2015) The nod-like receptor, Nlrp12, plays an anti-inflammatory role in experimental autoimmune encephalomyelitis. J Neuroinflammation 12: 198-211.

34. Oh H, Ghosh S (2013) NF-кB: Roles and regulation in different $\mathrm{CD}_{4}(+)$ T-cell subsets. Immunol Rev 252(1): 4151.

35. Katsara M, Yuriev E, Ramsland PA, Tselios T, Deraos G, et al. (2009) Altered peptide ligands of myelin basic protein (MBP87-99) conjugated to reduced mannan modulate immune responses in mice. Immunology 128(4): 521533.

36. Fitzsimmons REB, Mazurek MS, Soos A, Simmons CA (2018) Mesenchymal stromal/stem cells in regenerative medicine and tissue engineering. Stem Cells Int 8031718.

37. Cheng W, Zhao Q, Xi Y, Li C, Xu Y, et al. (2015) IFN- $\beta$ inhibits $\mathrm{T}$ cells accumulation in the central nervous system by reducing the expression and activity of chemokines in experimental autoimmune encephalomyelitis. Mol Immunol 64(1): 152-162.

38. Bai L, Lennon DP, Eaton V, Maier K, Caplan A, et al. (2009) Human bone marrow-derived mesenchymal stem cells induce Th2-polarized immune response and promote endogenous repair in animal models of multiple sclerosis. Glia 57(11): 1192-1203.

39. Liu X, Leung S, Wang C, Zhu T, Tan Z, et al. (2010) Crucial role of interleukin-7 in $\mathrm{T}$ helper type 17 survival and expansion in autoimmune disease. Nat Med 16(2): 191197.

40. Ashbaugh JJ, Brambilla R, Karmally SA, Cabello C, Malek TR, et al. (2013) IL7R $\alpha$ contributes to experimental autoimmune encephalomyelitis through altered $\mathrm{T}$ cell responses and nonhematopoietic cell lineages. J
Immunol 190(9): 4525-4534.

41. Sheng W, Yang F, Zhou Y, Yang H, Low PY, et al. (2014) STAT5 programs a distinct subset of GM-CSF-producing $\mathrm{T}$ helper cells that is essential for autoimmune neuroinflammation. Cell Res 24(12): 1387-1402.

42. Dunham J, Lee LF, Driel NV, Laman JD, Ni I, et al. (2016) Blockade of CD127 exerts a dichotomous clinical effect in marmoset experimental autoimmune encephalomyelitis. J Neuroimmune Pharmacol 11(1): 73-83.

43. Lawson BR, Gonzalez-Quintial R, Eleftheriadis $T$, Farrar MA, Miller SD, et al. (2015) Interleukin-7 is required for $\mathrm{CD} 4(+) \mathrm{T}$ cell activation and autoimmune neuroinflammation. Clin Immunol 161(2): 260-269.

44. Su N, Shi SX, Zhu X, Borazanci, A, Shi FD, et al. (2014) Interleukin-7 expression and its effect on natural killer cells in patients with multiple sclerosis. J Neuroimmunol 276(1-2): 180-186.

45. Lundström W, Highfill S, Walsh STR, Beq S, Morse E, et al. (2013) Soluble IL7R $\alpha$ potentiates IL-7 bioactivity and promotes autoimmunity. Proc Natl Acad Sci USA 110(19): 1761-1770.

46. Lee LF, Axtell R, Tu GH, Logronio K, Dilley J, et al. (2011) IL-7 promotes TH1 cell differentiation and its level in serum predicts response to interferon- $\beta$ in multiple sclerosis. Sci Transl Med 3(93): 93ra68.

47. Nuro-Gyina PK, Rieser EL, Granitto MC, Pei W, Liu Y, et al. (2016) Regulation of effector function of CNS autoreactive CD4 $\mathrm{T}$ cells through inhibitory receptors and IL-7R $\alpha$. J Neuroinflammation 13: 302.

48. Walline CC, Kanakasabai S, Bright JJ (2011) IL-7R $\alpha$ confers susceptibility to experimental autoimmune encephalomyelitis. Genes Immun 12(1): 1-14.

49. Axtell RC, De Jong BA, Boniface K, Van der Voort LF, Bhat $\mathrm{R}$, et al. (2010) $\mathrm{T}$ helper type 1 and 17 cells determine efficacy of IFN- $\beta$ in multiple sclerosis and experimental encephalomyelitis. Nat Med 16(4): 406-412.

50. Goverman J (2009) Autoimmune T cell responses in the central nervous system. Nat Rev Immunol 9(6): 393-407.

51. Ding X, Cao F, Cui L, Ciric B, Zhang GX, et al. (2015) IL-9 signaling affects central nervous system resident cells during inflammatory stimuli. Exp Mol Pathol 99(3): 570574.

52. Stephens GL, Swerdlow B, Benjamin E, Coyle AJ, Humbles AA, et al. (2011) IL-9 is a Th17-derived cytokine that limits pathogenic activity in organ-specific autoimmune 
disease. Eur J Immuol 41(4): 952-962.

53. Voet S, Printz M, Loo GV (2019) Microglia in central nervous system inflammation and multiple sclerosis pathology. Trends in Mol Med 25(2): 112-123.

54. Jäger A, Dardalhon V, Sobel RA, Bettelli E, Kuchroo VK (2009) Th1, Th17, and Th9 effector cells induce experimental autoimmune encephalomyelitis with different pathological phenotypes. J Immunol 183(11): 7169-7177.

55. Yin jj, Hu XQ, Mao ZF, Bao J, Qiu W, et al. (2017) Neutralization of interleukin-9 decreasing mast cells infiltration in experimental autoimmune encephalomyelitis. Chinese Med J 130(8): 964-971.

56. Li H, Nourbakhsh B, Cullimore M, Zhang GX, Rostami A (2011) IL-9 is important for T-cell activation and differentiation in autoimmune inflammation of the central nervous system. Eur J Immunol 41(8): 21972206.

57. Pandiyan P, Yang XP, Saravanamuthu SS, Zheng L, Ishihara S, et al. (2012) The role of IL-15 in activating STAT5 and fine-tuning IL-17A production in CD4 T lymphocytes. J Immunol 189(9): 4237-4246.

58. Zozulya AL, Wiendl H (2008) The role of regulatory $\mathrm{T}$ cells in multiple sclerosis. Nature Clin Practice Neurol 4: 384-398.

59. Zhao L, Hu B, Zhang Y, Song Y, Lin D, et al. (2016) An activation-induced IL-15 isoform is a natural antagonist for IL-15 function. Sci Rep 6: 25822.

60. Harrigan JA, Jacq X, Martin NM, Jackson SP (2018) Deubiquitylating enzymes and drug discovery: emerging opportunities. Nature Rev Drug Discov 17: 57-78.

61. Richmond JM, Strassner JP, Zapata L, Garg M (2018) Antibody blockade of IL-15 signaling has the potential to durably reverse vitiligo. Science Transl Med 10(45): 7710 .
62. Yu P, Bamford RN, Waldmann TA (2014) IL-15-dependent $\mathrm{CD} 8+\mathrm{CD}_{122}+\mathrm{T}$ cells ameliorate experimental autoimmune encephalomyelitis by modulating IL-17 production by CD4+ T cells. Eur J Immunol 44(11): 3330-3341.

63. Suto A, Kashiwakuma D, Kagami SI, Hirose K, Watanabe $\mathrm{N}$, et al. (2008) Development and characterization of IL-21-producing CD4+ T cells. J Exp Med 205(6): 13691379.

64. Lee Y, Mitsdoerffer M, Xiao S, Gu G, Sobel RA (2015) IL$21 \mathrm{R}$ signaling is critical for induction of spontaneous experimental autoimmune encephalomyelitis. J Clin Invest 125(11): 4011-4020.

65. Raveney BJE, Oki S, Yamamura T (2013) Nuclear receptor NR4A2 orchestrates Th17 cell-mediated autoimmune inflammation via IL-21 signalling. PLoS One 8(2): e56595.

66. Liu R, Bai Y, Vollmer TL, Bai XF, Jee Y, et al. (2008) IL21 receptor expression determines the temporal phases of experimental autoimmune encephalomyelitis. Exp Neurol 211(1): 14-24.

67. McGinley AM, Edwards SC, Raverdeau M, Mills KHG (2018) Th17 cells, $\gamma \delta$ T cells and their interplay in EAE and multiple sclerosis. J Autoimmun 87: 97-108.

68. Andersson A, Isaksson $\mathrm{M}$, Wefer J, Norling A, FloresMorales A, et al. (2008) Impaired autoimmune $\mathrm{T}$ helper 17 cell responses following DNA vaccination against rat experimental autoimmune encephalomyelitis. PLoS One 3(11): e3682.

69. Sashinami H, Asano K, Yoshimura S, Mori F, Wakabayashi $\mathrm{K}$, et al. (2012) Salmon proteoglycan suppresses progression of mouse experimental autoimmune encephalomyelitis via regulation of Th17 and Foxp3(+) regulatory T cells. Life Sci 91(25-26): 1263-1269.

70. Kang Y, Zhao J, Liu Y, Chen A (2009) FK506 as an adjuvant of tolerogenic DNA vaccination for the prevention of experimental autoimmune encephalomyelitis. J Gene Med 11(11): 1064-1070. 